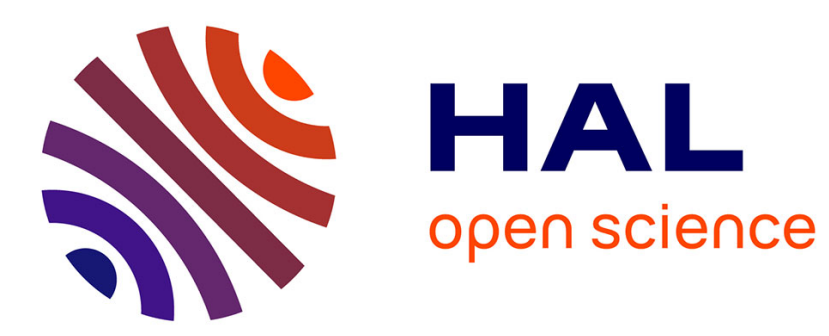

\title{
Histoire et acculturation chez les indiens Ixil du Guatemala
}

Pierre Becquelin

\section{To cite this version:}

Pierre Becquelin. Histoire et acculturation chez les indiens Ixil du Guatemala. Journal de la Société des américanistes, 1970, 59, pp.7-26. 10.3406/jsa.1970.2062 . hal-02932989

\section{HAL Id: hal-02932989 \\ https://hal.science/hal-02932989}

Submitted on 8 Sep 2020

HAL is a multi-disciplinary open access archive for the deposit and dissemination of scientific research documents, whether they are published or not. The documents may come from teaching and research institutions in France or abroad, or from public or private research centers.
L'archive ouverte pluridisciplinaire HAL, est destinée au dépôt et à la diffusion de documents scientifiques de niveau recherche, publiés ou non, émanant des établissements d'enseignement et de recherche français ou étrangers, des laboratoires publics ou privés. 


\section{Histoire et acculturation chez les indiens Ixil du Guatemala}

Pierre Becquelin

\section{Citer ce document / Cite this document :}

Becquelin Pierre. Histoire et acculturation chez les indiens Ixil du Guatemala. In: Journal de la Société des Américanistes. Tome 59, 1970. pp. 7-26;

doi : https://doi.org/10.3406/jsa.1970.2062

https://www.persee.fr/doc/jsa_0037-9174_1970_num_59_1_2062

Fichier pdf généré le 29/03/2019 


\title{
HISTOIRE ET ACCULTURATION CHEZ LES INDIENS IXIL DU GUATEMALA
}

\author{
par Pierre BECQUelin
}

Les Ixil occupent la région de Nebaj, située sur le versant septentrional des Cuchumatanes, dans les chaînes du Nord des Hautes Terres du Guatemala. Au Sud la région est séparée de la dépression sèche de Sacapulas par une barrière montagneuse dont l'altitude maximum est de 3349 mètres. De là le versant descend vers les Basses Terres du Petén en une série de crêtes est-ouest d'altitude décroissante, coupées de petites dépressions et de profondes vallées en gorge. Le réseau hydrographique se compose des bassins supérieurs des Ríos Xacbal et Cotzal. Climat et végétation changent graduellement en fonction de l'altitude : après une petite zone de prairie andine on entre vers 3000 mètres dans la zone de la forêt humide de montagne, qui s'enrichit d'espèces tropicales au fur et à mesure de la descente en zone chaude, atteinte vers 800 mètres d'altitude près de la limite nord de la région.

La population indigène parle l'ixil, langue du groupe maméen de la famille linguistique maya. L'ethnologie de la région est surtout connue par les travaux de Lincoln $(1942,1945)$, interrompus par sa mort en cours de mission. On sait grâce à lui que les communautés ixil des trois villages de la région, Nebaj, Cotzal et Chajul, sont parmi les plus riches du Guatemala en traits préhispaniques. En particulier on trouve chez les Ixil des prêtres utilisant les anciens calendriers cérémoniel et solaire et pratiquant des observations astronomiques.

L'archéologie de la région révèle une évolution culturelle complexe qui commence au deuxième siècle de notre ère (période protoclassique) par l'arrivée des premiers groupes d'agriculteurs porteurs des traditions des Hautes Terres. Au cours de la période classique (de 250 à 1000) les habitants de la région sont fortement influencés par la civilisation maya des Basses Terres, et le grand centre cérémoniel de Nebaj connaît alors son apo- 
gée. La présence de terrains de jeu de balle, de tombes à voûte en encorbellement, de stèles aniconiques, de céramique polychrome et de bijoux de jade témoigne de relations étroites avec les Basses Terres et de l'existence d'une société très hiérarchisée, sans doute organisée selon le modèle maya classique. La période postclassique (de 1000 à 1530) marque un repli : après l'effondrement de la civilisation maya des Basses Terres, la région de Nebaj ne connaît plus qu'un réseau de petits centres adaptés à une topographie fragmentée et à des communications difficiles. Cette structure de l'habitat correspond à un morcellement politique. Les influences extérieures sont ressenties avec moins d'intensité ; en particulier les contacts avec les grands centres protohistoriques fondés par les Quichés et les Cakchiquels, dont l'élite dirigeante prétend descendre des Toltèques du Mexique central, semblent avoir été de faible conséquence. A la veille de la conquête espagnole la région de Nebaj partage avec les autres régions des Hautes Terres certaines traditions céramiques, mais son architecture et son urbanisme conservent la plupart des traits anciens datant de la fin de la période classique.

On possède peu de documents historiques concernant la région de Nebaj. Il n'existe pas de textes indigènes, aucun dictionnaire établi par les religieux n'est parvenu jusqu'à nous ${ }^{1}$ et les chroniqueurs espagnols ne s'étendent guère sur l'histoire des Ixil. Cependant nous tenterons de donner une idée générale de l'évolution qui s'est accomplie entre le moment de la conquête et l'époque contemporaine, dans le but de mettre en valeur les survivances préhispaniques et de définir les grandes étapes de l'acculturation.

\section{PÉRIODE PROTOHISTORIQUE}

On désigne généralement sous ce terme la période postclassique récente (de 1250 à la conquête espagnole), en raison de l'existence de manuscrits quichés et cakchiquels dont les données s'ajoutent à celles de l'archéologie. Mais ces manuscrits ne mentionnent jamais les Ixil ${ }^{2}$. Les manuscrits quichés décrivent de nombreuses interventions armées dans la région de Sacapulas (centre anciennement nommé en quiché Tuhal), y compris sur la rive gauche du Río Negro (nom local du Río Chixoy), mais le récit de campagne le plus complet (Título Real de Don Francisco Izquin Nehaib, in Recinos

1. On ignore le sort d'un "Vocabulario de las Lenguas Ixil, Cacchi de Coban y de San Miguel Chicah " cité par Brasseur de Bourbourg (1857-59, tome I : 87).

2. Deux noms identiques à des noms de lieu de la région de Nebaj apparaissent dans ces textes : Acul comme nom de personne et nom de tribu (Recinos 1957:29, 31, 63 et Recinos 1950 : 171), Chipal comme nom de lieu sous la forme Hacavitz-Chipal (Recinos et Goetz $1953: 172-73$ ). Le contexte ne permet pas de relier ces Acul à la zone ixil ; quant à Hacovitz-Chipal, Recinos indique que Brasseur de Bourbourg sịtuait ce lieu près de Rabinal. 


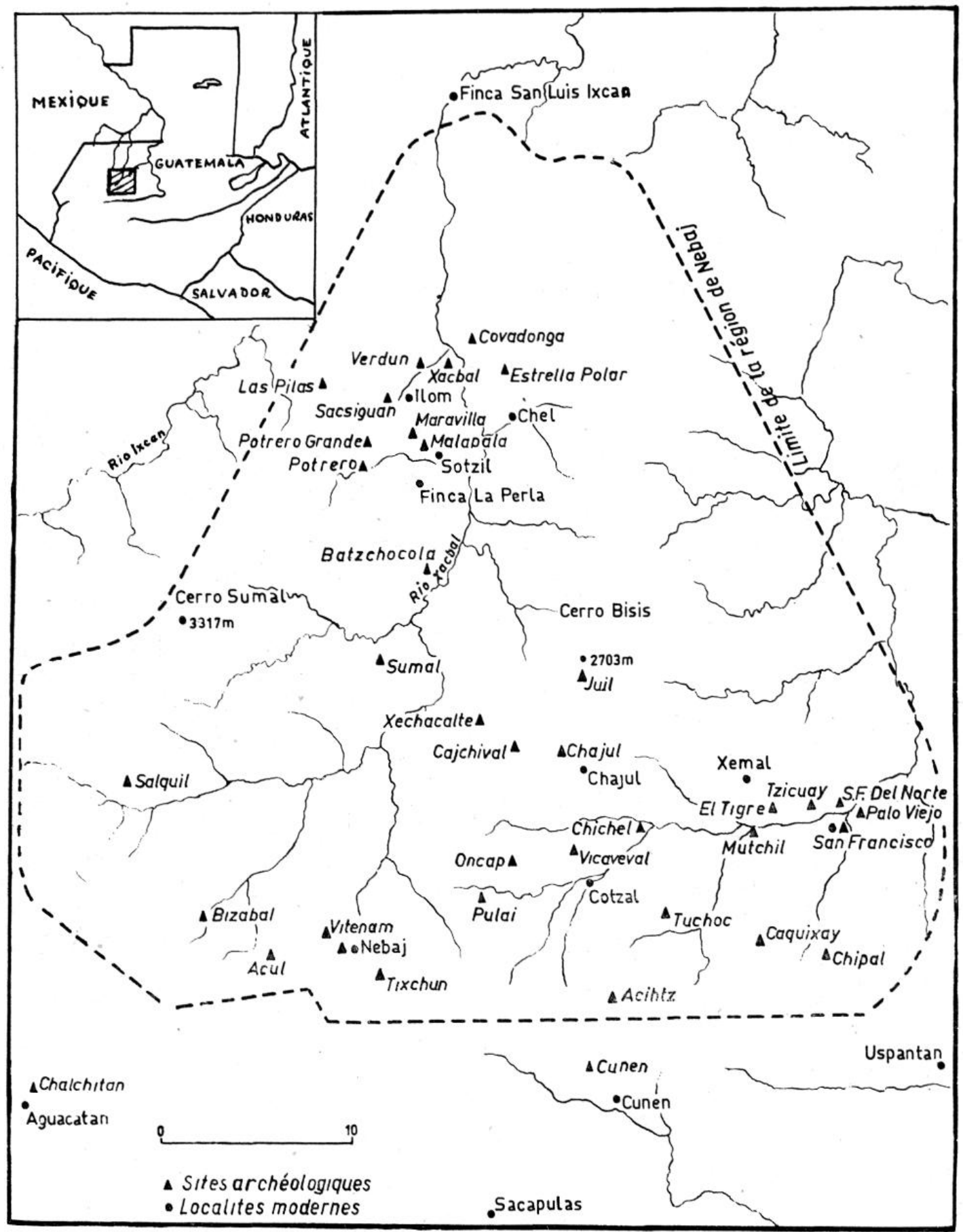

Fig. 1. - Carte de la région de Nebaj. 
1957 : 95-117) n'indique aucune pénétration dans les montagnes ixil. Les Quichés de Utatlán semblent avoir contrôlé fermement la région de Sacapulas puisqu'ils utilisaient leurs prisonniers de guerre à en exploiter les salines (Scholes et Adams 1960 : 218). Il se peut qu'antérieurement les Ixil soient intervenus dans la région, car les " títulos territoriales » d'Aguacatán, village situé à l'Ouest de Sacapulas, font allusion à l'abandon d'un centre nommé Lamac (aujourd'hui Salinas Magdalena, un peu moins de $5 \mathrm{~km}$ au N.-O. de Sacapulas) à la suite de guerres contre les gens des montagnes et autres événements désastreux (Elgueta 1899 : 38).

Le seul texte indigène où l'on puisse trouver des indications sur la région ixil est celui du ballet-drame Rabinal Achi, recueilli par Brasseur de Bourbourg en 1855 dans le village de Rabinal, habité par des indigènes de langue achi, du groupe linguistique quichéen. Ce spectacle se compose de musique, danses et dialogues relatant un épisode des guerres entre gens de Rabinal et Quichés de Utatlán, au cours duquel un prince quiché est fait prisonnier puis sacrifié ${ }^{1}$. Ce prince est présenté comme faisant partie du clan principal des Quichés, celui des Cavek, et porte entre autres titres celui de " ahau ri Yaqui Cunén, Yaqui Chajul "(Brasseur de Bourbourg 1862 : 34), ce qui signifie " chef des étrangers de Cunén, des étrangers de Chajul " (Raynaud $1953: 38)$. Ce titre semble indiquer qu'à la suite de conquêtes le prince a reçu le gouvernement de ces deux communautés. Cunén est un village situé dans une petite vallée au Nord de la dépression de Sacapulas; il a été fondé par les Dominicains qui y ont regroupé des indigènes de langue quiché. Chajul a été fondé dans les mêmes conditions : c'est le plus au Nord des trois villages ixil ${ }^{2}$. Si le texte du Rabinal-Achi ne contient pas ici d'anachronismes et que ces deux centres, Cunén et Chajul, existaient bien avant la conquête, on doit conclure qu'à une certaine période les Quichés ont exercé leur domination sur toute la partie est de la région de Nebaj.

Il est difficile de donner une date aux événements qui ont fourni l'argument du ballet-drame de Rabinal. Le prince quiché est dit être " $u$ qahol Balam-Achi, Balam-Quiché ri rahaual Quiché vinak "(Brasseur de Bourbourg 1862 : 24-25), c'est-à-dire " fils de Balam-Achi, Balam-Quiché chef des Quichés ». Balam-Quiché, dont le nom signifie Jaguar Quiché, est l'un des quatre chefs légendaires des Quichés. Mais ce nom peut également désigner un chef historique, puisque dans le "Título de los Señores de Totonicapan " (Recinos et Goetz $1953: 179-80$ ) il est dit que les titres et noms des grands ancêtres peuvent être portés par leurs successeurs. Dans ce cas, étant donné que le texte du Rabinal Achi mentionne par ailleurs Iximché, le grand centre cakchiquel fondé vers 1470 , il est possible que les conquêtes et guerres auxquelles il est fait allusion aient eu lieu entre cette date et celle de la

1. A propos de la possibilité plus que douteuse de représentations du Rabinal Achi dans la région de Nebaj, voir Termer $1957: 208$.

2. La carte de Fuentes y Guzmán (1933, tome $3: 89$ ) plaçant Chajul dans la dépression de Sacapulas est totalement inexacte. 
conquête espagnole ${ }^{1}$. Quant à la nature exacte des interventions quichés dans la région de Nebaj, on peut la déduire de l'absence de population quiché dans la région au moment de la conquête et du conservatisme marqué révélé par les données archéologiques : il s'agissait très probablement d'expéditions ayant pour but d'obtenir paiement d'un tribut en signe de soumission, ce qui n'implique pas l'existence de contacts importants.

On voit que les textes indigènes nous renseignent peu sur la protohistoire des Ixil. L'archéologie permet quelques inférences : on a déjà cité la faiblesse des influences extérieures et le morcellement politique. L'aspect des sites change peu : il s'agit toujours de petits centres cérémoniels comprenant plusieurs édifices sur substructures, généralement groupés autour d'un terrain de jeu de balle. D'après ce que l'on sait du reste du Guatemala, ces centres étaient la résidence des lignages nobles. On verra que les documents espagnols permettent de préciser quelle était la structure de l'habitat.

\section{PÉRIODE DE L'ACCULTURATION INITIALE}

Cette période est celle de la conquête et de l'évangélisation et s'étend de 1529 à 1600.

On trouve le récit des campagnes qui aboutirent à la soumission de la région de Nebaj dans les deux chapitres de la "Recordación Florida " consacrés par Fuentes y Guzmán à la "guerra de Uspantlan " (1933, tome 3 : 58-64). La conquête fut suivie du regroupement des indigènes en trois villages et de leur évangélisation. On peut distinguer dans les événements trois sous-périodes :

- 1529-30, conquête militaire.

- 1530-1553, soumission sans acculturation notable.

- 1553-1600, conquête spirituelle et acculturation majeure.

La conquête de la région ixil n'a été entreprise que lorsque la domination espagnole était déjà fermement établie sur la plus grande partie du Guatemala. Alvarado en 1524 soumet les Quichés de Utatlán, les Zutuhil, les Pipil de la côte du Pacifique. En 1525 c'est au tour des Mam de Zaculeu d'être soumis. En 1528 les Espagnols triomphent définitivement de la révolte des Cakchiquels. C'est alors que la décision est prise d'intervenir contre les indiens de Uspantán et leurs alliés ixil, dont l'aggressivité risquait de provoquer des troubles chez les Quichés déjà soumis ${ }^{2}$. La première campagne est

1. Lorsque Lincoln (1945) avance la date approximative de 1200 ap. J.-C. pour le règne de Balam-Quiché, c'est en commettant l'erreur de considérer une généalogie légendaire comme historique.

2. Termer (1958: 159) donne pour motif de l'intervention l'aide apportée par les Ixil 
dirigée par Gaspar Arias, avec 60 fantassins espagnols et 300 indiens. En six mois (jusqu'en septembre 1529) il obtient la reddition de plusieurs centres indigènes, dont Nebaj et Chajul. Ayant dû laisser le commandement à Pedro de Olmos, ce dernier lance sur Uspantán un assaut désastreux qui se termine en déroute. Les Espagnols commencent aussitôt une seconde campagne, sous la direction de Francisco de Castellanos, avec 40 fantassins, 32 cavaliers et 400 indiens, surtout mexicains et tlaxcaltèques. Les effectifs engagés sont faibles car d'autres combats sont en cours contre les Chorti d'Esquipulas et les Pipil de Cuzcatlan. Castellanos se dirige vers Nebaj après avoir franchi le Rio Negro sur un pont provisoire aux environs de Sacapulas. Il livre deux combats autour des passages de la crête, contre 4 à 5000 guerriers (les gens de Nebaj et leurs alliés), puis descend sur le centre indigène qu'il prend d'assaut. Les principaux chefs sont capturés ; les prisonniers sont réduits en esclavage et marqués au fer. A la suite de cette victoire Chajul fait aussitôt sa reddition. A la fin du mois de décembre de cette même année 1530 Castellanos prend Uspantán, malgré l'aide apportée à ce centre par ses alliés de Cotzal (Ixil) et de Cunén et Sacapulas (Quichés). Là encore les prisonniers sont réduits en esclavage.

Uspantán est actuellement un village situé à l'Est de Cunén, dans une vallée de la zone faillée qui prolonge la dépression de Sacapulas. Les Dominicains y ont regroupé au $\mathrm{xvI}^{\mathrm{e}}$ siècle la population de langue uspantèque, du groupe linguistique quichéen. Plusieurs sites archéologiques sont signalés aux environs du village, l'un d'eux étant vraisemblablement l'ancien centre indigène. Comme en témoigne le récit de Fuentes y Guzmán, Uspantán dirigeait la coalition qui s'opposait aux conquérants. Cette prépondérance régionale, qui s'étendait à la région de Sacapulas antérieurement soumise aux Quichés de Utatlan, était probablement la conséquence de la défaite de ces derniers.

Entre 1530 et 1553, date de la fondation du couvent de Sacapulas, nous ne savons presque rien de la région de Nebaj. Il semble qu'elle soit restée soumise, sans nouvelle intervention militaire espagnole. Des Franciscains ont visité la zone montagneuse de Sacapulas (Remesal 1932, tome $2: 332$; Vásquez, 1937-44, tome $2: 32,35)$ et probablement la région de Nebaj, car ils se plaignent dans un texte de 1552 des expéditions guerrières annuelles des Lacandons venus des Basses Terres (Saint-Lu 1968 : 283). La culture préhispanique devait être encore bien vivante. Les coutumes funéraires en tout cas n'avaient pas changé : dans une tombe du centre cérémoniel de Chipal on a trouvé un vase espagnol déposé comme offrande en même temps que d'autres vases et objets de style protohistorique (Butler 1956, 1959).

Pendant ce temps la conquête des régions voisines progressait avec la sou-

aux Mam de Zaculeu. Rien ne permet d'identifier l'armée de gens des montagnes (“ ejército de serranos ", in Fuentes y Guzmán 1933, tome $3: 123$ ) comme Ixil. Il s'agit bien plutôt d'indigènes des Altos Cuchumatanes venus secourir les assiégés de Zaculeu. Même erreur d'identification dans Stoll (1960:60). 
mission pacifique, due aux efforts des Dominicains, de la Terre de Guerre, le Tesulutlan, qui devient la province de la Vera Paz. Les religieux procèdent aux premiers regroupements : fondation de Rabinal en 1542, puis de Coban en 1544. La couronne espagnole avait décidé par la Cédula Real du 10 juin 1540 de généraliser la méthode de la concentration de la population indigène, destinée à faciliter l'évangélisation rendue difficile par l'habitat rural dispersé. Rien n'est fait cependant dans la région de Nebaj avant la fondation du couvent dominicain de Sacapulas en 1553. On trouve dans Remesal (1932, tome $2: 332-334)$ quelques détails sur cette fondation. L'Audiencia établie à Guatemala envoie des lettres aux " caciques " indigènes pour les en aviser et leur ordonner d'aider les religieux. La liste de ces chefs nous donne des indications sur la géographie politique des régions de Nebaj, Sacapulas et zones voisines. La lettre du président de la «Audiencia de los Confines " s'adresse aux chefs suivants : caciques de Zacapula (Sacapulas), Aguacatlan (Aguacatán), Zaquila (Salquil), Zacabaha (Sajcabajá), Huil, Ylon (Ilóm) et Chacoa. La lettre de l'Auditeur Zorita s'adresse aux caciques de Chalchutlan (Chalchitán), Balamihá ${ }^{1}$, Nebah (Nebaj), Acul, Chaxá, Cuneb (Cunén) et Valancolob (Balamcolob) ${ }^{2}$.

Ce qui permet d'établir la liste suivante :

a) Noms correspondant à ceux de villages actuels : Sacapulas, Aguacatán, Sajcabajá, Nebaj, Cunén.

b) Noms correspondant à ceux de hameaux actuels : Salquil, Acul (commune de Nebaj), Huil, Ilóm, Chaxá (commune de Chajul).

c) Autres catégories : noms de populations mal identifiées (Balamihá, Balamcolob), nom d'un quartier actuel d'Aguacatán (Chalchitán) ${ }^{3}$.

Signalons que certains des villages fondés par les Dominicains de Sacapulas ne figurent d'aucune manière dans ces listes : Chajul, Cotzal, Uspantán, Jocotenango. Imprécision administrative?

Après l'envoi des lettres aux caciques le 6 décembre 1553, le couvent est construit grâce au travail indigène. Juan de Torres en est nommé prieur. A la demande des religieux Pedro Ramírez de Quiñonez, de la «Audiencia de los Confines " 4 , ordonne le regroupement des Ixil en trois villages. Dans

1. Balamihá : nom de l'une des tribus ayant participé à la migration légendaire des Quichés selon le Popol Vuh (Recinos 1950 : 171). Dans le Título de los Señores de Totonicapan la tribu est citée sous le nom de Ahcab-Balamiha (Recinos et Goetz $1953: 188$ ). Le Título de los Indios de Santa Clara la Laguna cite les Agab y Balimaha parmi les tribus contre lesquelles les Quichés doivent défendre leurs frontières (Recinos 1957 : 179). D'après Carmack (1967 : 5) Balamija est le nom quiché des Aguacatèques.

2. Balamcolob : nom de l'une des tribus ayant participé à la migration légendaire des Quichés (Recinos 1950 : 171).

3. Chalchitán : nom du quartier est d'Aguacatán, village fondé par les Dominicains. Ce village est divisé en deux quartiers, Aguacatán proprement dit et Chalchitán.

4. Ramírez de Quiñonez était l'un des trois juges de l'Audiencia et en fut président en 1558-59. Nous ignorons la date exacte de l'ordre de regroupement. 
Remesal $(1932$, tome $2: 245)$ on trouve les noms de quelques-uns des centres préhispaniques regroupés ${ }^{1}$, dont certains peuvent être identifiés soit à des sites archéologiques soit à des subdivisions communales actuelles (" aldeas ", c'est-à-dire hameaux, ou " caserios ", groupes de maisons).

A Chajul ont été regroupés les habitants de Huyl, Boob, Ylom, Honcab, Chaxa, Aguazaq, Huiz et quatre autres centres dont le nom n'est pas donné. On peut identifier :

- Huyl : Huil, site archéologique et caserio du hameau Chacalté.

- Ylom : Ilóm, hameau.

- Chaxá : caserio du hameau Xemal.

- Honcab : Oncap, site archéologique, ou Joncab, caserio de Chajul voisin du site. Lincoln (1945) identifie Honcab avec Xoncá, hameau de Nebaj.

- Huiz : identifié par Lincoln $(1942,1945)$ mais sans en préciser la localisation, que nous n'avons pu déterminer.

A Nebaj ont été regroupés les habitants de Vaca, Chel, Zalchil, Cuchil et plus de douze autres centres. On peut identifier :

- Zalchil : Salquil, site archéologique et hameau de Nebaj.

- Chel : hameau de Chajul ; ce hameau très éloigné de Nebaj ne correspond probablement pas au centre cité par Remesal ${ }^{2}$.

A Cotzal ont été rassemblés les indigènes de Nama, Chicui, Temal, Caquilax et de nombreux autres centres. On peut identifier les centres suivants :

- Namá : caserio de Asich, hameau de Cotzal et site archéologique.

- Chicui : peut-être Tzicuay, site archéologique et caserio du hameau de Chichel.

On retrouve parmi les noms de ces centres ceux des centres cités précédemment comme étant dirigés par un cacique, à l'exception de Acul. En ce qui concerne les trois villages actuels le texte de Remesal implique nettement l'existence de Nebaj et Cotzal avant les regroupements, le cas de Chajul étant moins clair.

En donnant la liste partielle des centres dont les habitants ont été rassemblés à Chajul, Remesal ajoute que chacun d'eux avait autorité sur d'autres centres plus petits : " y cada uno destos tenía otros pueblezuelos conjuntos como sufragáneos " (1932, tome $2: 245)$. Il semble donc que le réseau des centres se présentait ainsi :

— Centres dirigés par un chef important ("cacique ») : Nebaj, Acul, Salquil, Huil, Ilóm, Chaxá.

1. La population rurale vivait dispersée autour des centres cérémoniels habités par l'élite dirigeante.

2. Chel est peut-être cité ici par erreur, au lieu d'être mentionné à propos de Chajul. 
- Centres moyens, peut-être subordonnés aux précédents.

- Petits centres dépendants des deux catégories précédentes.

La liste des noms de centres dressée d'après Remesal correspond assez peu à celle des sites archéologiques connus. Seulement quatre centres peuvent être identifiés à des sites occupés au Postclassique récent : Acul, Salquil, Huil et probablement Oncap. Les trois premiers étaient occupés par un " cacique ". Dans les trois cas il s'agit d'un petit centre cérémoniel du type courant dans la région, ne se distinguant pas des douze autres centres connus à la même période. L'autorité politique ne s'accompagnait pas d'une élaboration particulière de l'habitat. Quant aux petits centres (" pueblezuelos ") ils correspondent aux groupes d'habitation, plus difficiles à découvrir par l'archéologue car ne possédant pas de substructures importantes et de bâtiments cérémoniels.

Avec la fondation des trois villages qui permet de concentrer la population peut commencer vraiment la conquête spirituelle. Nous ne possédons pas de données précises sur ses modalités dans la région de Nebaj, mais il est certain que les Dominicains y ont employé leurs méthodes habituelles et ont réussi à modifier profondément la culture indigène. Comme partout ailleurs, les villages sont organisés selon un plan orthogonal centré sur une place rectangulaire que domine l'église. Les divinités indigènes doivent disparaître et les cérémonies indigènes sont remplacées par les fêtes chrétiennes. L'évangélisation s'accompagne de la formation de " cofradias " (confréries de dévotion). L'organisation politique indigène fait place à des municipalités que contrôlent les religieux et l'administration coloniale. Le surplus économique auparavant utilisé par les chefs et prêtres indigènes est désormais destiné au paiement du tribut (depuis 1550), de la dîme et des contributions versées aux caisses de communauté administrées par les religieux ou selon leurs directives. Un champ de maïs dont la récolte va au prêtre doit être cultivé par les indiens (Colby et Van den Berghe 1969 : 59). De plus les indigènes sont soumis à l'exploitation économique des conquérants laïques : Colby et Van den Berghe (1969: 47) signalent la présence de deux encomiendas ${ }^{1}$ dans la région ixil, dont une à Chajul sans date connue et une dans la région de Nebaj, sans précision géographique, quand Francisco Díaz del Castillo était corregidor de Totonicapan, c'est-à-dire vers 1579 (Recinos 1954 : 505). Une encomienda est mentionnée comme ancienne et vacante pour cause de décès dans un document de 1677 ; elle consistait en l'héritage en deuxième génération du droit aux tributs de Cotzal et Joyabaj (SaintLu 1968 : 226, note 128). Depuis 1549 l'encomienda était limitée à la réception d'un tribut, sans droit de résidence de l'encomendero. Les encomiendas furent abolies définitivement au Guatemala après 1720 (Recinos $1954: 214$ ). Dans la région de Nebaj la législation dut être respectée car les encomiendas

1. Encomienda : ensemble de droits et de devoirs reconnus à des conquistadors sur des communautés indiennes. 
ne donnèrent pas naissance à des haciendas privées. L'exploitation agricole de cette zone montagneuse éloignée n'intéressait sans doute pas les Espagnols. De nombreux éléments de la culture matérielle sont également modifiés. La maison à toit de tuile date de cette période. Les religieux introduisent les outils de fer, dont le sabre d'abattage (" machete »), qui deviendra l'indispensable outil à tout faire, ainsi que de nouveaux cultigènes et animaux domestiques, dont les animaux de bât. Ces améliorations peuvent être considérées comme secondaires au regard des conséquences de la disparition de la classe dirigeante indigène, disparition qui privait les communautés ixil de toute possibilité de conduite indépendante en matière de politique et de religion.

En 1570 les Dominicains entreprennent la construction du pont de Sacapulas franchissant le Río Negro. Ils distribuent aux villages voisins leur tâche : chacun doit construire une pile et une arche. Le pays indien semble se transformer peu à peu en terre chrétienne. Mais à cette date commence, en Amérique centrale comme au Mexique, une période d'affaiblissement de l'effort missionnaire au cours des trente dernières années du $\mathbf{x v I}^{\mathrm{e}}$ siècle, pour aboutir au siècle suivant à un abandon quasi général de la tentative de conquête spirituelle.

On peut s'étonner de ce qu'une aussi courte période d'évangélisation intense ait pu provoquer tant de changements dans la culture indigène. On doit tenir compte de l'ébranlement des valeurs traditionnelles provoqué par la conquête, créant ainsi une atmosphère favorable à l'acceptation de nouvelles certitudes. Mais il ne faut pas oublier que l'effort acharné des religieux utopiques s'est exercé sur une ou deux générations indiennes très diminuées par de fréquentes épidémies. En 1530 on peut estimer la population indigène à 16000 ou 20000 habitants, en prenant pour base le nombre de guerriers indiqué par le chroniqueur espagnol. A la fin du xvir siècle l'administration espagnole ne peut recenser que 1916 habitants indigènes pour toute la région de Nebaj (Fuentes y Guzmán 1933, tome 3 : 57-58). Cette diminution vertigineuse a frappé toute l'Amérique indigène, où l'on estime que la population a subi un taux de réduction de 20 à 1 en 130 ans, de 1519 à 1650 (Dobyns 1966).

Cet effondrement démographique a facilité la tâche des religieux, avant que ses conséquences économiques ne viennent, au $\mathrm{xvII}^{\mathrm{e}}$ siècle, ruiner les rêves de richesse des conquistadors.

\section{PÉRIODE INTERMÉDIAIRE OU D'AJUSTEMENT}

Dans l'ensemble des Hautes Terres, à l'exception de leur partie orientale, cette période est celle de la culture indigène traditionnelle. $L$ ' " indigène traditionnel " est né lors de la création dans les nouveaux villages d'une culture composée des traits préhispaniques qui ont persisté et des traits 
nouveaux introduits principalement par les missionnaires. Cette longue période est marquée par des contacts fréquents mais limités avec le clergé et l'administration coloniale puis nationale. La notion d'indigène traditionnel est nouvelle : le terme a pour fonction de marquer le contraste avec l' " indigène modifié ", produit d'une acculturation plus avancée (R.N. Adams 1964 : 24-25).

Cette période s'étend de 1600 à 1887, date de l'installation dans la région de Nebaj des premiers "ladinos " ${ }^{1}$.

La culture indigène traditionnelle conserve les éléments introduits par les missionnaires au cours de la première période. Un certain nombre de ces éléments sont réinterprétés, par exemple dans le rituel des confréries, l'accent mis sur le culte des saints, le vêtement caractéristique de chaque village, les danses. Certains éléments préhispaniques sont conservés : calendrier annuel et cérémoniel indigène interprété par des prêtres spécialisés, divinisation des noms de jour et en particulier des quatre jours du calendrier cérémoniel par lesquels peut commencer l'année solaire, ces jours étant associés aux quatre directions de l'espace, caractère sacré des montagnes (en particulier le Mont Sumal), procédés de divination, médecine liée à l'usage du bain de vapeur, degré élevé de monolinguisme.

Faute dé témoignages suffisants sur cette période nous ne pouvons aborder en détail que certaines questions : l'efficacité des regroupements, les contacts entre Ixil et indiens insoumis (les Lacandons), l'influence du clergé catholique, l'économie de la région et les modalités de l'imposition.

Le but des Dominicains était de concentrer toute la population en trois villages. Il se heurtait aux habitudes des indigènes et aux données de la géographie, le territoire des communes nouvelles étant fort étendu. Si les religieux parvinrent à réunir dans les villages un certain nombre d'habitants, les documents ultérieurs n'en attestent pas moins la persistance d'une forte dispersion.

Lincoln (1945 : 47-48) cite une note écrite probablement au milieu du xıx $^{\mathrm{e}}$ siècle par le père Baltasar Valdivia, curé de Nebaj, sur les pages de couverture du plus ancien registre de baptêmes conservé à Chajul et couvrant les années 1676-1778. On y apprend que Ilóm, l'un des centres préhispaniques cité par Remesal, fut abandonné à une date indéterminée après l'évangélisation : les livres de l'église furent envoyés à Soloma, l'autel à Chajul et les cloches à Santa Eulalia ${ }^{2}$. Dans une autre note, écrite cette fois dans le livre paroissial de Nebaj couvrant les années 1835-53, Valdivia précise que les gens de Ilóm émigrèrent en deux groupes qui s'établirent l'un à

1. Le terme ladino s'applique à tout individu n'appartenant pas à une communauté indigène. Généralement il s'agit d'un métis dont la culture est d'origine hispanique. (R. N. Adams 1964 : 20-21).

2. Santa Eulalia et Soloma sont deux villages des Altos Cuchumatanes, situés à environ $35 \mathrm{~km}$ en ligne directe de Ilóm. Les indiens de Santa Eulalia parlent kanjobal et ceux de Soloma, solomèque. 
Chajul et l'autre à Santa-Eulalia. Ce dernier dut être assimilé entièrement car il n'a pas laissé de traces linguistiques. Lorsque Lincoln visita Sotzil en 1939 un fonctionnaire indigène lui déclara que l'objet le plus sacré de Santa Eulalia, le Holom Konop (tête du village), pièce de bois qui est sans doute une statue érodée, avait été apportée de Ilóm, ce qui confirme la migration. Le même informateur plaça la fondation de l'actuel Sotzil 150 ans auparavant, soit vers 1790. Lorsque Termer visita Ilóm en 1927, douze ans avant Lincoln, on lui indiqua que la fondation de ce hameau remontait à 150 ans (Termer $1957: 9$ ). On lui dit aussi que le hameau de Chel avait été fondé à la même époque, c'est-à-dire vers 1775 . En réalité la fondation est plus ancienne puisque Ilóm figure dans une liste de tributs de 1752 (Colby et Van den Berghe $1969: 60$ ).

On peut reconstituer ainsi les événements : bien que les religieux aient tenté d'obtenir le maximum de concentration de la population, ils furent obligés de consentir au maintien de certains petits centres, dont Ilóm. Valdivia dans le livre paroissial de Nebaj énumère d'autres hameaux où furent édifiées de petites églises : Salquil, Acul, Chichel (hameaux actuels), Ahcana (peut-être Jacana, caserío du hameau Xonca) et Magdalena (sans doute Salinas Magdalena, l'ancien Lamac). Puis Ilóm fut évacué, selon les termes de Valdivia, " pour éviter que les néophytes de Ilóm continuent de fréquenter les forêts et solitudes immenses des territoires du Río de la Pasión au confluent avec le Río de Chixoy ou Lacantun ". On craignait sans doute le contact avec les indiens Lacandons. Au milieu du xvirI ${ }^{e}$ siècle de nouveaux hameaux furent fondés à Sotzil, Ilóm et Chel. Dans ce cas précis les habitudes indigènes se révélèrent finalement plus fortes que les tentatives de modification. Notons cependant que ces hameaux sont concentrés. La permanence d'une certaine dispersion est par ailleurs attestée par d'autres textes : selon Fuentes y Guzmán les habitants de Nebaj sont à la fin du $\mathrm{xvII}^{\mathrm{e}}$ siècle répartis en un grand nombre de hameaux, "Cuchil, Salquil, Yghil et autres " (1933, tome $3: 57)$. Cortés y Larraz (1958, tome $2: 48-49$ ) note lors de sa visite de 1769 que les indigènes de la région de Nebaj préfèrent vivre dans les montagnes où ils peuvent échapper au paiement $d u$ tribut. Ces habitudes rendaient le contrôle administratif difficile et une Cédula Real de 1579 demandait déjà à l'alcalde mayor de Totonicapan de recenser les indiens de Chajul qui échappaient au paiement du tribut (Elliot 1966).

La structure actuelle de l'habitat, définie par la juxtaposition d'un habitat rural concentré et d'un habitat rural dispersé dominant, résulte d'un compromis entre tendances indigènes et européennes certainement déjà réalisé à la fin du xviri ${ }^{\mathbf{e}}$ siècle. Il s'agit là d'un processus très général : en 1577 une Cédula Real enjoignait à l'Audiencia de Guatemala de veiller à ce que les indigènes ne reviennent pas habiter leurs anciens centres (Remesal 1932, tome 2 : 247). L'habitat dispersé n'en persista pas moins, la plupart des indiens continuant d'habiter près de leurs champs, autour de leurs anciens temples ruinés. 
D'autres difficultés risquaient de nuire à la domination espagnole. Au Nord de la zone ixil, dans les Basses Terres, vivaient les Lacandons insoumis, qui parfois faisaient des incursions dans les villages. Les appréciations concernant les rapports entre Lacandons et indigènes soumis varient beaucoup selon les auteurs. Nous avons vu que les Franciscains signalaient des attaques annuelles. Fuentes y Guzmán (1933, tome $3: 58$ et 86-88) déclare que les Lacandons commercent avec les villages des Altos Cuchumatanes et procèdent parfois à des attaques. Des déplacements de population ont dû ètre effectués afin d'éloigner le plus possible des Lacandons les indigènes christianisés de Santa Eulalia et San Mateo Ixtatán. Les habitants de Cotzal et Cunén auraient été également en rapport avec les Lacandons. Tovilla, qui fut alcalde mayor de La Vera Paz, visita Chajul en 1631 et y recueillit le récit d'un indigène qui avait été enlevé à l'âge de neuf ans lors d'une attaque de Lacandons, et devenu adulte était parvenu à retourner à son village (Scholes et Adams 1960 : 208-11). On peut relever plusieurs détails intéressants : le danger était si fréquent que l'on confia aux gens de Chajul douze arquebuses ; les Lacandons sacrifiaient leurs prisonniers ; l'enfant capturé fut épargné en tant que parent de l'un des Lacandons; Tovilla put l'interroger grâce à la présence d'un vieil Ixil parlant Lacandon ; enfin pour regagner son village le prisonnier dut marcher quatorze jours : il est vrai qu'il ignorait la plus grande partie de l'itinéraire. Tout cela nous suggère des contacts assez fréquents, variant de l'alliance à l'hostilité, entre populations qu'une assez grande distance séparait. Les attaques avaient pour but de se procurer des prisonniers pour les sacrifices religieux.

Ximenez, dans sa chronique rédigée entre 1715 et 1722 (1930, tome 2 : 220-22), considère que le danger lacandon a été exagéré et que le nombre des attaques réelles est peu élevé. Il cite une incursion de 1664 au cours de laquelle les Lacandons s'emparèrent d'un enfant de Chajul et le sacrifièrent en procédant à l'ablation du cœur. L'attaque s'était produite dans un champ de maïs situé à quatre lieues (soit plus de seize kilomètres) du village ${ }^{1}$. Il mentionne également l'enlèvement $d^{\prime}$ 'un indigène de Chajul qui parvint ensuite à regagner son village lorsqu'il atteignit l'âge adulte : il s'agit là sans doute de l'incident déjà décrit par Tovilla. Après l'occupation par les Espagnols en $1695 \mathrm{du}$ village lacandon le plus proche de la région de Nebaj ${ }^{2}$, tout péril avait disparu. Pourtant Ximenez cite une fausse alerte à Cotzal en 1720, au cours de laquelle tout le village s'enfuit, abandonnant le religieux qui s'y trouvait en visite. On continua longtemps encore de croire à un danger

1. Termer (1957: 8) pense avec vraisemblance que les Lacandons effectuaient leurs incursions en remontant le Río Xacbal. Il en déduit que la frontière des Ixil était située à peu près dans la zone attaquée en 1664 , sans tenir compte des textes qui prouvent l'existence de Ilóm au moment de la conquête.

2. Ce village nommé Dolores par les Espagnols était habité par des Lacandons de langue chol. Il était probablement situé entre l'actuelle frontière mexicaine et le Río Usumacinta (Thompson 1938). 
devenu légendaire, puisqu'on signala à Cortés y Larraz au cours de sa visite à Nebaj la présence de Lacandons dans les montagnes entre Chajul et Soloma ; mais l'archevêque déclare qu'il a de bonnes raisons de penser qu'il s'agit en réalité d'indigènes qui ont quitté leurs villages et se tiennent à l'écart de l'administration espagnole (Cortés y Larraz 1958, tome 2 : 48).

Dans la lutte entre foi nouvelle et anciennes traditions, l'influence des indigènes restés insoumis a joué un rôle négligeable. Bien plus grandes ont été les conséquences du maintien dans les anciens lieux d'habitat. Mais ce facteur même n'a dû son importance qu'à la faiblesse de l'encadrement religieux ${ }^{1}$.

En 1617 il n'y avait au couvent de Sacapulas que huit Dominicains ayant la charge de treize villages (Remesal 1932, tome $2: 611$ ). Dans la région ixil seul Nebaj était une paroisse avec un prêtre résident, Chajul et Cotzal n'étant que des annexes (Fuentes y Guzmán 1933, tome $3: 57$ ). L'archevêque Cortés y Larraz constate à Nebaj l'absence d'école, note qu'il faudrait un prêtre par village. Lors de son passage le curé était remplacé par un Dominicain qui avait les plus grandes difficultés à maîtriser la langue ixil. La coutume était de faire fouetter les indigènes qui n'assistaient pas à la messe (Cortés y Larraz 1958, tome $2: 45-50)$. Au début du $\mathrm{xrx}^{\mathrm{e}}$ siècle la paroisse était toujours administrée par les Dominicains, dans le cadre des exceptions à la sécularisation de 1754 (Juarros 1808-18:72). Les prêtres n'avaient pas renoncé à tout progrès de l'évangélisation : sans doute en raison des difficultés dues à l'ignorance persistante de l'espagnol par les indiens, un curé de Nebaj rédigea vers 1824 un petit ouvrage intitulé "Doctrine et confession en langue ixil précédées d'une brève méthode pour apprendre la langue et le rituel du mariage "(Doctrina 1824). D'autres prêtres sont plus découragés ; le curé Valdivia écrit vers 1850 : "Que les missionnaires apostoliques de ces populations supportent le fait qu'après trois cents ans d'évangélisation elles se trouvent en pire état qu'au premier siècle régressant vers la vieille barbarie mêlée des vices et de l'irréligion des autres castes "(Lincoln 1945).

Si la conquête et ses suites n'avaient pas abouti à faire des Ixil de bons chrétiens, l'élimination d'une grande partie des traditions indigènes était au moins certaine. Et le contrôle religieux et administratif, joint à l'exploitation économique, rendait impossible l'existence d'une élite ixil. Les membres des municipalités indigènes étaient considérés par l'administration comme des intermédiaires devant assurer le paiement de diverses formes d'imposition qui pesaient lourdement sur une population demeurée au stade de l'économie de subsistance.

Les rapports (" relaciones ") soumis au gouvernement espagnol par les alcaldes mayores chargés de l'administration de la région, qui fait partie

1. Il n'existait pas d'encadrement civil : aucun document ne mentionne de fonctionnaires ou soldats espagnols résidant dans les villages ixil. 
du Partido de Huehuetenango dépendant de la Alcaldía Mayor de Totonicapan, permettent de juger de la situation économique. Un rapport de 1740 (Olavarreta 1935) indique une forte production de maïs, beaucoup d'arbres fruitiers, des mules. On fait des travaux de vannerie à Chajul et de corderie à Cotzal où l'on utilise le moulinet d'origine espagnole ; les femmes de Chajul tissent beaucoup. Un rapport de 1765 (Urbina 1937) déclare plus brièvement que toute l'alcaldía mayor est au niveau de l'économie de subsistance et que le commerce y est nul. Pourtant on tente d'en tirer le maximum. D'abord par le paiement du tribut, difficile à assurer : il est arrivé que les alcaldes fussent maintenus en prison vingt-sept mois pour non-paiement (Fuentes y Guzmán 1933, tome 2 : 58). Ensuite la main-mise sur les « cajas de comunidad ", administrées par la Real Hacienda à partir de 1639. En outre chaque alcalde mayor impose aux villages des "repartimientos ": on oblige les indigènes à recevoir du coton brut qu'ils doivent rendre filé deux fois par an, ou à acheter au prix fort des houes, ou plus rarement à livrer certaines quantités de maïs ${ }^{1}$.

Avec la fin de la domination espagnole en 1821 la désorganisation temporaire de l'administration a pu soulager momentanément les communautés indigènes. Mais le nouvel état indépendant continua d'exploiter les indiens. Dès 1821-22 les fonds des caisses de communauté sont confisqués pour tenter de combler le déficit du budget. Un fait nouveau apparaît : au cours des luttes acharnées que se livrent conservateurs et libéraux après l'indépendance, les indigènes se trouvèrent parfois entraînés à prendre parti pour l'un ou l'autre camp. En 1869 les libéraux attaquèrent Huehuetenango. Au cours du combat la ville fut pillée et en partie incendiée. Les troupes libérales étaient accompagnées d'indiens d'Aguacatán, Nebaj, Cotzal, Chajul et Cunén (Recinos 1954 : 266-67). De telles péripéties ne modifiaient pas l'essentiel de la situation des indigènes, traités en catégorie inférieure sans être soumis à un processus direct d'acculturation.

Le seul progrès est démographique; au cours de la période d'ajustement la population indigène double :

$\begin{array}{lllll}\text { vers } 1700: 1 & 916 & \text { habitants indigènes } & \text { (Fuentes y Guzmán) } \\ 1769: 2909 & " & \text { " } & \text { (Cortés y Larraz) } \\ 1800: 3367 & \text { " } & \text { " } & \text { (Juarros) } \\ 1825: 4005 \quad " & " & \text { (Rivera Maestre) }\end{array}$

1. Pour les "repartimientos " dans la région de Nebaj, consulter Arrollave 1937, Guzman 1937, Recinos 1954 : 216. Pour la répression en cas de refus, voir Cortés y Larraz 1958, tome $2: 48-50$. 


\section{PÉRIODE DE L'ACCULTURATION NOUVELLE}

Avec l'arrivée des “ladinos » dans la région de Nebaj s'ouvre une nouvelle période d'acculturation, amorçant le passage de l'indigène traditionnel à l'indigène modifié.

Les possibilités légales de l'acquisition des terres indigènes par les ladinos ont été créées par les décrets du président Rufino Barrios en 1877. L'obligation de posséder un titre de propriété permettait de considérer comme vacants de nombreux terrains occupés par les indigènes, qui ne pouvaient fournir aucune preuve légale de leurs droits. L'essor de la production de café incita ladinos et étrangers à créer des plantations dans de nouvelles zones. La première ladina s'installa à Nebaj en 1887. En 1893 il y avait 98 ladinos dans la région. L'exploitation économique des indigènes et la création des " fincas " de café aboutirent parfois à des incidents violents (en 1925 à Ilóm, en 1936 à Nebaj). Une nouvelle acculturation est provoquée à la fois par le contact avec les ladinos locaux et par les déplacements d'indigènes vers la côte du Pacifique en tant que travailleurs agricoles saisonniers ${ }^{1}$. Ces migrations deviennent de plus en plus importantes en raison de l'explosion démographique :

\begin{tabular}{|c|c|c|}
\hline $1893: 12601$ & & \\
\hline $1921: 19.606$ & $"$ & $"$ \\
\hline $1950: 28218$ & $"$ & ") \\
\hline $1964: 45040$ & ” & $"$ \\
\hline
\end{tabular}

Depuis l'ouverture en 1945 d'une route reliant la région de Nebaj au reste du pays les transformations tendent à s'accélérer. Lorsqu'on arrive à Nebaj, on est toujours frappé de la permanence des traits traditionnels les plus apparents, par exemple le pourcentage élevé de monolinguisme, le vêtement distinctif et coloré des femmes et d'une partie des hommes, le tissage avec métier horizontal simple, les cérémonies des confréries, l'usage persistant du bain de vapeur. Mais il est certain que la transmission de nombreux traits culturels anciens est de plus en plus perturbée. Il s'agit d'une acculturation lente, bien différente $d u$ bouleversement apporté par la conquête et l'évangélisation.

\section{CONCLUSIONS}

Il est clair que les faits connus sont rares, souvent imprécis, et ne concernent que certains aspects de la société et de la culture ixil. Il est impossible d'étudier dans le détail les mécanismes de l'acculturation passée, et très

1. On trouvera la plupart des faits concernant cette période dans Lincoln (1945), et Colby et Van den Berghe (1969). 
difficile d'évaluer son ampleur exacte en raison de notre méconnaissance de la période antérieure à la conquête. Cependant les documents permettent d'étudier le rythme de cette acculturation et son résultat global, c'est-àdire de décider, compte tenu de l'état actuel des communautés indigènes, si la transformation a été assez profonde pour effacer les traces de l'état initial.

La Farge (1940) a présenté une séquence des cultures indigènes dont les données lui ont été fournies par ses travaux dans les Altos Cuchumatanes. Elle comprend cinq périodes : après la période de la conquête (1524-1600) il distingue une période indigène coloniale (1600-1720), une première transition marquée par la disparition de l'encomienda (1720-1800), puis une période indigène récente $I$ (1800-1880) marquée par un affaiblissement des interventions extérieures dans les communautés indigènes, et enfin une période indigène récente II, celle de l'acculturation nouvelle. Cette séquence a été adoptée, avec de légères modifications de formulation, par Beals et Villa qui la donnent pour également valable au Mexique (Heritage of Conquest, 1952, pp. 227-29 et 250,288 ), ce qui nécessite certaines modifications chronologiques en raison de la date plus tardive au Guatemala de faits cruciaux tels que la fin des encomiendas et des missions. Le point essentiel étant l'histoire de l'acculturation, il n'y a en fait que deux périodes fondamentales, celle de la modification initiale et celle de la modification récente. La période intermédiaire d'ajustement ne peut être subdivisée, en employant les catégories de La Farge, que lorsque l'on dispose d'un nombre suffisant de documents. Ce n'est pas le cas dans la région de Nebaj.

En ce qui concerne la survivance des traits préhispaniques, la région de Nebaj est l'une des plus conservatrices, de même que les Altos Cuchumatanes. Cependant ce qui subsiste est bien faible. Les opinions des anthropologues sont partagées sur l'ampleur des modifications, selon qu'ils considèrent l'ensemble des traits culturels ou portent l'accent sur la disparition de l'élite et des éléments les plus élaborés de la culture (Heritage of Conquest, 1952, pp. 253-54, 256-57, 290; Wolf 1959, pp. 211-13, 284-85). Dans ce dernier cas, il est évident que les Ixil, avec leur culture marginale et leurs petits centres cérémoniels, avaient relativement moins à perdre que les Quichés ou Cakchiquels dépendant de capitales telles que Utatlán et Iximché. Mais leur déculturation et la réduction de la complexité sociale, bien que moins notables, n'en ont pas moins été considérables en valeur absolue, d'autant plus que les pertes dues à l'intervention directe du clergé et de l'administration coloniale, à l'appauvrissement, à l'effondrement démographique étaient mal compensées par l'intégration difficile de traits culturels ibériques, d'ailleurs sélectionnés dans le cadre d'une culture de conquête (Foster 1960).

En dépit de tout, une nouvelle culture ixil s'est constituée. Même sous sa forme actuelle, l'acculturation n'a guère avancé vers l'assimilation, et en particulier l'ethos indigène n'a pas été éliminé. Il demeure orienté essentiellement vers les valeurs de la communauté. Mais en dépit des résistances, ce 
qui demeure de la culture indigène traditionnelle et des traits préhispaniques qu'elle a préservés ne peut sans doute, dans les conditions actuelles, que se dégrader dans la surpopulation ou se dissoudre dans la culture des ladinos, et dans ce cas sous la forme de celle des ouvriers agricoles les plus pauvres.

\section{REFERENCES}

Adams, R. N. (1964) : Encuesta sobre la cultura de los ladinos en Guatemala. Guatemala, Seminario de Integración Social Guatemalteca.

Arrollave, J. de (1935) : Relación Geográfica del Partido de Huehuetenango. Boletin del Archivo General del Gobierno, Tome I, No 1, pp. 16-24. Guatemala.

Beals, R. L. (1967) : Acculturation. In : Handbook of Middle American Indians, volume 6, pp. 449-468. Austin.

Becquelin, P. (1969) : Archéologie de la Région de Nebaj, Guatemala. Paris, Institut d'Ethnologie.

Brasseur de Bourbourg, C. E. (1857-59) : Histoire des Nations civilisées du Mexique et de l'Amérique Centrale. Paris.

Brasseur de Bourbourg, C. E. (1862) : Rabinal Achi ou le drame-ballet du Tun, in : Grammaire de la Langue Quichée..., Paris, A. Bertrand.

Butler, M. (1956) : The case of the Chipal pot. Philadelphia Anthropological Society Bulletin, Vol. 9, 2, pp. 11-12. Philadelphia.

Butler, M. (1959) : Spanish contact at Chipal, in : Amerikanistiche Miszellen, Mitteilungen aus dem Museum für Völkerkunde in Hamburg, Vol. XXV, pp. 28-35.

Carmack, R. M. (1967) : Analisis Histórico-Sociológico de un Antiguo Título Quiché. Antropología e Historia de Guatemala, Vol. XIX, no 1, pp. 3-13.

Colbý, B. N. et P. L. van den Berghe (1969) : Ixil Country : A Plural Society in Highland Guatemala. Berkeley, University of California Press.

Cortes y Larraz, P. (1958) : Descripción geográfico-moral de la Diócesis de Goathemala. Guatemala, Sociedad de Geografía e Historia, 2 vol.

Doвyns, H. F. (1966) : Estimating aboriginal american population. An appraisal of techniques with a new hemispheric estimate. Current Anthropology, Vol. 7, $\mathrm{n}^{0} 4$, pp. 395-416.

Doctrina y confesionario en lengua ixil (1824). Precedidos de un corto modo para aprender la lengua y Ritual de Matrimonio por el cura Parroco de Nebah. University Museum, Philadelphia.

Elgueta, M. G. (1899) : Etimolojías nacionales. Totonicapán, Tipografía popular.

Ellior, R. et H. (1966) : Ixil, in Lenguas de Guatemala, Seminario de Integración Social Guatemálteca, Guatemala, pp. 179-99.

Foster, G. M. (1960) : Culture and Conquest : America's Spanish Heritage. WennerGren Foundation for Anthropological Research.

Fuentes y Guzman, F. A. de (1932-33) : Recordación Florida. Guatemala, Sociedad de Geografía e Historia, 3 vol. 
Guzman, F. M. de (1937) : Auto formado sobre la Real Cédula... Boletín del Archivo General del Gobierno, Tome II, no 3, pp. 304-307. Guatemala.

Heritage of conquest (1952). The Free Press, Glencoe.

Juarros, D. (1808-18) : Compendio de la Historia de la Ciudad de Guatemala. Guatemala.

LA Farge, O. (1940) : Maya Ethnology : The Sequence of Cultures, in The Maya and their Neighbors, New York et Londres, pp. 281-91.

Lincoln, J.S. (1942) : The Maya Calendar of the Ixil of Guatemala. Contributions to American Anthropology and History, no 38, pp. 97-128. Carnegie Institution of Washington.

Lincoln, J. S. (1945) : An ethnological study of the Ixil indians of the Guatemala Highlands. Microfilm Collection of Manuscripts on Middle American Cultural Anthropology, $\mathrm{n}^{\circ}$ 1. Chicago, University of Chicago Library.

NASH, M. (1969) : Guatemalan Highlands. In : Handbook of Middle American Indians, volume 7, pp. 30-45. Austin.

Olavarreta, J. de (1935) : Relación Geográfica del Partido de Huehuetenango. Boletín del Archivo General del Gobierno, Tome I, no 1, pp. 16-24. Guatemala.

Raynaud, G. (1935) : Rabinal Achi, ballet-drama de los indios Quichés de Guatemala. Guatemala, Ministerio de Educación Pública.

Recinos, A. (1950) : Popol Vuh. The sacred book of the ancient Quiché Maya. Norman, University of Oklahoma Press.

Recrivos, A. (1954) : Monografía de Huehuetenango. Guatemala, Ministerio de Educación Pública.

Recinos, A. (1957) : Crónicas indígenas de Guatemala. Guatemala, Editorial Universitaria.

Recinos, A. et D. Goetz (1953) : The Annals of the Cakchiquels. Title of the Lords of Totonicapan. Norman, University of Oklahoma Press.

Remesal, A. de (1932) : Historia general de las Indias Occidentales y particular de la Gobernación de Chiapa y Guatemala. Sociedad de Geografía e Historia, Guatemala, 2 vol.

Rivera Maestre, M. (1832) : Atlas Guatemalteco en 8 cartas formadas y grabadas en Guatemala... Guatemala 1832.

Saint-Lu, A. (1968) : La Vera Paz. Esprit évangélique et colonisation. Paris, Centre de Recherches Hispaniques.

Scholes, F. V. et E. B. Adams (1960) : Relaciones histórico-descriptivas de la Verapaz, el Manché y Lacandón, en Guatemala. Guatemala, Editorial Universitaria.

Stoll, O. (1960) : Los indios ixiles. Boletin del Instituto Indigenista Nacional, Vol. II, pp. 59-64. Guatemala.

Termer, F. (1957) : Etnología y Etnografia de Guatemala. Guatemala, Seminario de Integración Social Guatemalteca.

Termer, F. (1958) : Apuntes geográficos y etnográficos de la zona de Nebaj. Anales de la Sociedad de Geografía e Historia de Guatemala, Vol. 31, pp. 150-66.

Tномpson, J. E. S. (1938) : Sixteenth and seventeenth century reports on the Chol Maya. American Anthropologist, Vol. 40, pp. 585-604.

Urbina, I. de (1937) : Auto formado sobre la Real Cédula... Boletín del Archivo General del Gobierno, Tome II, no 3, pp. 307-309. Guatemala. 
VAsquez, F. (1937-44) : Crónica de la Provincia del Santisimo Nombre de Jesús de Guatemala. Sociedad de Geografía e Historia, Guatemala, 4 vol.

Worf, E. (1959) : Sons of the Shaking Earth. University of Chicago Press, Chicago et Londres.

Ximenez, F. (1929-31) : Historia de la Provincia de San Vicente de Chiapa y Guatemala. Sociedad de Geografía e Historia, Guatemala, 3 vol. 